\title{
A retrospective study on the prognostic value of preoperative C-reactive protein to albumin ratio in patients with oral cavity squamous cell carcinoma
}

\author{
Ku-Hao Fang ${ }^{1}$, Chiahsuan Lai ${ }^{2}$, Cheng-Ming Hsu ${ }^{3}$, Ethan Huang ${ }^{3}$, Ming Shao Tsai ${ }^{3}$, Geng-He Chang ${ }^{3}$, Yi-Chan \\ Lee $^{4}$, Yao-Te Tsai ${ }^{\text {Corresp. } 3}$ \\ ${ }^{1}$ Department of Otorhinolaryngology-Head and Neck Surgery, Chang Gung Memorial Hospital, Taoyuan, Taiwan \\ 2 Department of Radiation Oncology, Chang Gung Memorial Hospital, Chiayi, Taiwan \\ 3 Department of Otorhinolaryngology-Head and Neck Surgery, Chang Gung Memorial Hospital, Chiayi, Taiwan \\ 4 Department of Otorhinolaryngology-Head and Neck Surgery, Chang Gung Memorial Hospital, Keelung, Taiwan \\ Corresponding Author: Yao-Te Tsai \\ Email address: yaote1215@gmail.com
}

Background. Although the C-reactive protein-to-albumin ratio (CAR) can predict poor outcomes in assorted cancers, its prognostic value in oral cavity squamous cell carcinoma (OSCC) remains unclear. We explored the value of preoperative CAR in predicting clinical outcomes in OSCC patients treated with radical surgery. Methods. All the recommended cutoff values were defined analyzing receiver operating characteristic curves or overall survival (OS). Dichotomization was performed on the basis of optimal CAR cutoff, and we compared the clinicopathological features between groups. Kaplan-Meier analysis was also performed to compare OS curves between the two groups. Univariate and multivariate analyses using the Cox proportional hazards model were conducted to find the clinical characteristics that were most closely correlated with disease free survival (DFS) and overall survival (OS). A nomogram incorporated CAR and several clinicopathological factors was established to predict prognosis and its accuracy was evaluated using concordance index (c-index). Results. In this retrospective study, a total of 326 patients with newly diagnosis of OSCC and received primary surgery between 2008 and 2017 were enrolled. Through the executed ROC curve analyses, the optimal CAR cutoff derived was 0.195 (area under the curve $=0.718, p<0.001$ ), with this cutoff exhibiting a discrimination ability superior to that of other inflammation-based prognostic scores after comparing the area under curves. Multivariate analysis demonstrated that CAR $(\geq 0.195 /<0.195)$ was associated with OS (hazard ratio 3.614; 95\% confidence interval 1.629-8.018; $p=0.002$ ) and DFS (hazard ratio 1.917; 95\% confidence interval 1.051-3.863; $p=0.029$ ). KaplanMeier analysis and log rank test revealed a significant difference in DFS and OS curves between patients with low CAR $(<0.195)$ and those with high CAR $(\geq 0.195$; both $p<$ 0.001 ) The c-index of the nomogram based on TNM system alone was 0.684 and could be 
increased to 0.801 if CAR and other clinicopathological factors were included.

Conclusions. Preoperative CAR could constitute an independent prognostic indicator for OS and DFS prediction in OSCC patients treated with curative surgery. The established nomogram that incorporated CAR and prognostic factors might increase the accuracy of prognostic prediction for patients with OSCC. 
1 A retrospective study on the prognostic value of preoperative C-

2 reactive protein to albumin ratio in patients with oral cavity

3 squamous cell carcinoma

4 Ku-Hao Fang ${ }^{1}$, Chiahsuan Lai $^{2}$, Cheng-Ming Hsu ${ }^{3}$, Ethan Huang ${ }^{3}$, Ming Shao Tsai ${ }^{3}$, Geng-He

5 Chang ${ }^{3}$, Yi-Chan Lee $^{4}$, Yao-Te Tsai ${ }^{3 *}$

6

71 Department of Otorhinolaryngology-Head and Neck Surgery, Chang Gung Memorial Hospital, Linkou, Taiwan

82 Department of Radiation Oncology, Chang Gung Memorial Hospital, Chiayi, Taiwan

93 Department of Otorhinolaryngology-Head and Neck Surgery, Chang Gung Memorial Hospital, Chiayi, Taiwan

104 Department of Otorhinolaryngology-Head and Neck Surgery, Chang Gung Memorial Hospital, Keelung, Taiwan

11

12 *Corresponding author

13 Yao-Te Tsai, MD

14 Department of Otolaryngology-Head and Neck Surgery, Chiayi Chang Gung Memorial Hospital, 15 Address: No.6, W. Sec., Jiapu Rd., Puzi City, Chiayi County 613, Taiwan (R.O.C.)

16 Phone: $+886-5-3621000$ ext. 3726

17 E-mail: yaote1215@gmail.com

18

19

20

21

22

23 ABSTRACT

Peer) reviewing PDF | (2020:02:45632:1:1:NEW 6 May 2020) 
24 Background. Although the C-reactive protein-to-albumin ratio (CAR) can predict poor

25 outcomes in assorted cancers, its prognostic value in oral cavity squamous cell carcinoma

26 (OSCC) remains unclear. We explored the value of preoperative CAR in predicting clinical

27 outcomes in OSCC patients treated with radical surgery.

28 Methods. All the recommended cutoff values were defined analyzing receiver operating

29 characteristic curves or overall survival (OS). Dichotomization was performed on the basis of

30 optimal CAR cutoff, and we compared the clinicopathological features between groups. Kaplan-

31 Meier analysis was also performed to compare OS curves between the two groups. Univariate

32 and multivariate analyses using the Cox proportional hazards model were conducted to find the

33 clinical characteristics that were most closely correlated with disease free survival (DFS) and

34 overall survival (OS). A nomogram incorporated CAR and several clinicopathological factors

35 was established to predict prognosis and its accuracy was evaluated using concordance index (c36 index).

37 Results. In this retrospective study, a total of 326 patients with newly diagnosis of OSCC and received primary surgery between 2008 and 2017 were enrolled. Through the executed ROC curve analyses, the optimal CAR cutoff derived was 0.195 (area under the curve $=0.718, p<$ 0.001), with this cutoff exhibiting a discrimination ability superior to that of other inflammationbased prognostic scores after comparing the area under curves. Multivariate analysis demonstrated that CAR $(\geq 0.195 /<0.195)$ was associated with OS (hazard ratio $3.614 ; 95 \%$ confidence interval 1.629-8.018; $\mathrm{p}=0.002$ ) and DFS (hazard ratio 1.917; 95\% confidence interval 1.051-3.863; $\mathrm{p}=0.029)$. Kaplan-Meier analysis and log rank test revealed a significant difference in DFS and OS curves between patients with low CAR $(<0.195)$ and those with high CAR $(\geq 0.195$; both $p<0.001)$. The c-index of the nomogram based on TNM system alone was 
$47 \quad 0.684$ and could be increased to 0.801 if CAR and other clinicopathological factors were 48 included.

49 Conclusions. Preoperative CAR could constitute an independent prognostic indicator for OS and

50 DFS prediction in OSCC patients treated with curative surgery. The established nomogram that

51 incorporated CAR and prognostic factors might increase the accuracy of prognostic prediction

52 for patients with OSCC.

53

54 Keywords C-reactive protein-to-albumin ratio, oral cavity squamous cell carcinoma,

55 inflammation-based prognostic scores, prognostic predictor, nomogram 


\section{INTRODUCTION}

58 Despite the recent decline in betel quid use, the oral cavity squamous cell carcinoma (OSCC)

59 incidence has continued to increase in Taiwan (Liao et al., 2014); this increase may be explained

60 by the long-term carcinogenic effect engendered by betel quid chewing and by the detrimental

61 effects engendered by alcohol consumption and cigarette smoking (Adel et al., 2016). People's

62 cigarette smoking, betel quid chewing, and alcohol consumption not only cause field

63 cancerization but are also correlated significantly with systemic inflammation (Oliveira,

64 Rodriguez-Artalejo, \& Lopes, 2010, Shafique et al., 2012, Zhang et al., 2016). Some of the

65 available systemic inflammation indices involve patient-related factors; these indices include

66 pretreatment C-reactive protein (CRP) levels, platelet-to-lymphocyte ratio (PLR), neutrophil-to-

67 lymphocyte ratio (NLR), as well as modified Glasgow prognostic score (mGPS) and can be

68 applied as survival predictors in patients with various cancers (Brown et al., 2007, Crumley et al.,

69 2006, Read et al., 2006), including head and neck cancer (Takenaka et al., 2018a, 2018b). Of

70 these, the pretreatment mGPS, a score combining CRP and serum albumin levels, better predicts

71 cancer survival compared with the peripheral blood cell count-based prognostic scores (e.g.,

72 NLR and PLR) (Dutta et al., 2011). Recent studies have indicated that CRP-to-albumin ratio

73 (CAR), also created on serum albumin levels and CRP levels, is a valuable prognosticator in

74 various cancers and may provide more accurate prognostic prediction than other indicators

75 (Ishizuka et al., 2016). Because patients with OSCC frequently experience malnutrition and

76 inflammation due to eating disability and immunosuppression, CAR may serve as a novel

77 prognostic indicator in OSCC (Gellrich et al., 2015). However, the prognostic value achieved by

78 CAR in OSCC requires comprehensive examination, and a few studies with relatively small

79 patient numbers have probed this ratio's prognostic value in OSCC patients (Park, Kim, \& Kim, 
80

87

88

89

2016). Herein, in patients with OSCC treated with curative intent, we examined the prognostic significance of the following preoperative systemic inflammatory indices : CAR, mGPS, PLR, and NLR, highlighting the correlation of CAR with clinicopathological characteristics and treatment outcomes. In addition, a nomogram model incorporating CAR, sex, age, TNM staging, extracapsular nodal extension (ENE), depth of invasion (DOI), and cancer cell differentiation was established to predict the 3- and 5-year overall survival (OS) for patients with OSCC after curative surgical treatment.

MATERIALS AND METHODS

\section{Study patients}

We retrospectively reviewed the clinical outcomes of patients newly diagnosed with OSCC and who underwent primary curative surgery with or without adjuvant therapy at the Department of Otorhinolaryngology of Chang Gung Memorial Hospital from January 2008 until December 2017.

We subsequently excluded patients with a history of malignancy, synchronous cancer, infection or inflammatory conditions, or autoimmune disorders; those who had received neoadjuvant therapy; and those with missing CRP or albumin data. Finally, 326 patients were enrolled. Their history of cigarette smoking, betel nut chewing, and alcohol consumption was obtained from clinic notes and patient interviews or from the tumor registry. Cigarette smokers were defined as those who had smoked 1 or more cigarettes per day for 1 year or longer; betel nut chewers were defined as those who had chewed 2 betel nuts or more daily for at least 1 year; and alcohol drinkers were defined as those who had consumed more than 1 alcoholic beverage per week for more than 6 months. The Institutional Review Board of Chang Gung Memorial Hospital ratified 
103 our study protocol (201901573B0), and the requirement for patient's informed consent was

104 waived by the Institutional Review Board. All patients were subjected to routine preoperative

105 workups including blood tests, physical examinations, magnetic resonance imaging or computed

106 tomography of the head and neck, abdominal echography, chest X-ray, nuclear bone scans, and

107 the detailed medical histories of the patients were recorded. Concurrent neck dissection and

108 intraoperative frozen section controls were used for tumor excision per institutional guidelines,

109 and plastic surgeons used local, free, or pedicled flaps for reconstruction of surgical defects.

110 Pathological TNM staging was recorded according to the AJCC (American Joint Committee on

111 Cancer) Cancer Staging Manual Cancer Staging Manual, Eighth Edition. If indicated,

112 postoperative adjuvant therapy based on the institutional guidelines was administered. Briefly,

113 patients with pathological T4 tumors who had positive lymph nodes underwent adjuvant

114 radiotherapy, and patients with any pathological finding including multiple neck lymph node

115 metastases, positive surgical margin, and ENE received adjuvant concurrent chemoradiotherapy

116 within 6 weeks after surgery. A radiation dose of 66 Gy was administered in 2-Gy daily fractions

117 for 5 days each week, and a cisplatin-based regimen was used for the chemotherapy. Patients

118 were followed up bimonthly for the first year after discharge, at 3-month intervals throughout the

119 second year and at 6-month intervals thereafter. At each follow-up visit, physical examination,

120 laboratory testing, and endoscopy were performed. Follow-up imaging with computed

121 tomography or magnetic resonance imaging was performed every 6 months for 2 years and every

12212 months thereafter.

123

124 Inflammation-based prognostic scores

125 To probe the correlation between survival outcomes and systemic inflammatory indices, we

126 performed preoperative blood laboratory tests within 1 week before curative surgery. According 
127 to the blood tests and recorded clinical symptoms and signs, severe infection status was excluded

128 in all patients. The medical staff collected the hematological and biochemistrical parameters

129 during the treatment from patient's charts. Pretreatment biochemistry values of albumin

130 (reference value: $35-55 \mathrm{~g} / \mathrm{L}$ ) and CRP (reference value: $<5 \mathrm{mg} / \mathrm{L}$ ) were measured using

131 biochemistry automated analyzer (Roche Hitachi Cobas 8000, Rotkreuz, Switzerland) during the

132 study period. Hematological results of lymphocyte, neutrophil, hemoglobin, and platelet were

133 measured using the hematology analyzer (Sysmex SE-9000, Kobe, Japan). Preoperative CAR

134 was calculated as follows: CRP level (expressed in $\mathrm{mg} / \mathrm{L}$ )/albumin level (expressed in $\mathrm{g} / \mathrm{L}$ ).

135 Similarly, we derived the preoperative NLR and PLR as follows: peripheral blood neutrophil

136 count/lymphocyte count and platelet count/lymphocyte count, respectively. Next, mGPS was

137 calculated using previously published methods (McMillan, 2008). Patients with both

138 hypoalbuminemia ( $<35 \mathrm{~g} / \mathrm{L})$ and increased CRP levels $(>10 \mathrm{mg} / \mathrm{L})$, with one of these variables,

139 and with none of these variables were assigned the scores of 2 , 1 , and 0 , respectively.

140

141 Statistical analysis

142 By analyzing the receiver operating characteristic (ROC) curves, we determined the statistically

143 optimal cutoff values of prognostic variables on the basis of inflammation, including the CAR,

144 CRP, mGPS, PLR, and NLR. The area under the ROC curve (AUC) was calculated for

145 determining the various indices' discriminatory ability. We used the Spearman test to investigate

146 the correlation between preoperative CRP and albumin levels and examined the normality of

147 distribution of study data by using Kolmogorov-Smirnov test. Patient follow-up was conducted

148 at the outpatient clinic until death or the cutoff date (December 31, 2018). The log-rank test and

149 Kaplan-Meier analysis were performed to evaluate the long-term survival probability in the high

150 and low CAR groups (defined by the optimal CAR cutoff). The clinicopathological features of 
151 the two groups were compared using the Mann-Whitney $U$ and chi-square tests for continuous

152 and categorical variables, respectively. Cox proportional hazards models based univariate and

153 multivariate analyses were used to identify independent prognostic factors by calculating their

154 hazard ratios (HRs) and the corresponding 95\% confidence intervals (CIs). All aforementioned

155 analyses were performed on SPSS version 21.0 (SPSS, Chicago, IL, USA). Statistical

156 significance was indicated by $p<0.05$. A multivariate nomogram model incorporating sex, age,

157 overall pathological stage, cell differentiation, ENE, DOI, and preoperative CAR was generated

158 as described by Kao et al. (2018) by using the "rms" package in R (version 5.1-0, Vanderbilt

159 University, Nashville, TN, USA). Calibration plots were drawn and a concordance index (c-

160 index) of the established nomogram was calculated to assess the predictive accuracy for OS. A c-

161 index of 0.5 denotes the equivalent of random prediction and that of 1.0 indicates perfect

162 prediction (Harrell, Lee, \& Mark, 1996). We calculated the c-index for traditional OS prediction

163 based on TNM staging alone as well as for prediction with the proposed nomogram models with

164 and without CAR.

165

166 RESULTS

167 Baseline characteristics

168 Baseline clinicopathological characteristics as well as laboratory data are listed in Table 1. In

169 total, 326 patients - $294(90.2 \%)$ of whom were men and $32(9.8 \%)$ were women — who were

170 newly diagnosed as having OSCC and underwent primary radical surgery were included; their

171 median age and follow-up duration were 57 (range, 31-86) years and 48 (range, 3-115) months,

172 respectively. The most common primary tumor site was the tongue $(n=126,38.7 \%)$, followed

173 by the buccal area $(n=104,31.9 \%)$ and gingiva $(n=43,13.2 \%)$. Of these patients, $81.9 \%$ were 
174 smokers, $79.8 \%$ were betel nut chewers, and $66 \%$ were alcohol consumers. Nearly half of the

175 patients $(\mathrm{n}=152,46.6 \%)$ were diagnosed as having stage IV disease, and $117(35.8 \%)$ patients

176 had pathologically confirmed neck lymph node metastasis. All the enrolled patients completed

177 the planned treatment course, with 43 (13.2\%) patients undergoing only adjuvant radiotherapy

178 and $105(32.2 \%)$ receiving adjuvant concurrent chemoradiotherapy.

179

180 Inflammation-based prognostic score cutoffs and ROC curves

181 The median CAR was 0.08 (range, 0.01-5.48). A Spearman test revealed that CRP levels were 182 negatively correlated with albumin levels ( $r=-0.223 ; p<0.001$, Fig. 1$)$. By analyzing the ROC 183 curves, we determined the optimal OS cutoff value to be 0.195 for CAR (sensitivity, $65.3 \%$; 184 specificity, 78.4\%), 4.505 for NLR, and 165.85 for PLR. We further compared the AUCs of 185 various indices (Fig. 2) to assess their discrimination ability and found that the AUC of CAR $186(0.718,95 \%$ CI $[0.654-0.782], p<0.001)$ was higher than that of NLR $(0.621,95 \%$ CI $[0.550-$

$1870.692], p=0.001), \mathrm{CRP}(0.705,95 \% \mathrm{CI}[0.638-0.766], p=0.001), \mathrm{PLR}(0.610,95 \%$ CI $[0.539-$ 188 $0.680], p=0.002)$, and mGPS $(0.679,95 \%$ CI [0.612-0.746], $p<0.001)$.

189

190

\section{Association of CAR with clinicopathological characteristics}

191 We performed dichotomization of patients by the optimal cutoff of CAR, followed by comparing

192 the two groups' clinicopathological characteristics (Table 2). The CAR $\geq 0.195$ group was

193 determined to have a significant association with advanced TNM stage $(p<0.001)$, lymph node

194 metastasis with extracapsular nodal extension (ENE, $p<0.001)$, DOI of $>10 \mathrm{~mm}(p<0.001)$,

195 need for adjuvant therapy $(p=0.003)$, higher mGPS $(p<0.001)$, higher NLR $(p<0.001)$, higher

$196 \operatorname{PLR}(p=0.034)$, and shortened survival period $(p=0.01)$ compared with the other group. 


\section{Association of CAR with survival outcomes}

199 In the univariate analysis, the indicators of poor OS were found to be increased T classification,

200 lymph node metastasis with ENE, poor cell differentiation, DOI $>10 \mathrm{~mm}$, need for adjuvant

201 chemoradiotherapy, mGPS of 1 or 2 , CAR of $\geq 0.195$, NLR of $\geq 4.505$, and PLR of $\geq 165.85$

202 (Table 3). Furthermore, multivariate analysis indicated increased T classification ( $p=0.025$ for

$203 \mathrm{~T} 3$ and 0.003 for T4), ENE ( $p<0.001)$, poor cell differentiation $(p<0.001)$, CAR of $\geq 0.195(p=$

2040.002 ), and NLR of $\geq 4.505$ ( $p=0.006)$ to be independent prognostic factors for poor OS (Table

205 3). In the OS probability analysis, the observed 5-year OS incidence was $80.9 \%$ and $46.5 \%$ in

206 patients with an optimal CAR cutoff of $<0.195$ and $\geq 0.195$, respectively; these survival

207 differences were significant according to the executed log-rank test ( $p<0.001$, Fig. 3A). The

208 median OS times for patients with CARs of $\geq 0.195$ and $<0.195$ were 40 (95\% CI [12-68]) and

$209>99$ months, respectively.

210 Table 4 demonstrates the association of clinicopathological variables with 5-year DFS. In the

211 univariate analysis, T4 classification, ENE, poor cell differentiation, DOI of $>10 \mathrm{~mm}$, need for

212 adjuvant chemoradiotherapy, mGPS of 1 or 2 , CAR of $\geq 0.195$, NLR of $\geq 4.505$, and PLR of

$213 \geq 165.85$ were significantly associated with poor DFS. Multivariate analysis results indicated that

214 T4 classification $(p=0.031)$, ENE $(p<0.001)$, poor cell differentiation $(p=0.009)$, CAR of

$215 \geq 0.195(p=0.029)$, and NLR of $\geq 4.505(p=0.02)$ were independent prognostic indicators of

216 poor DFS. In the DFS probability analysis, the 5-year DFS incidence for patients stratified into

217 the $\mathrm{CAR}<0.195$ and $\mathrm{CAR} \geq 0.195$ subgroups was at a proportion of $60.1 \%$ and $36.8 \%$,

218 respectively; moreover, the log-rank test results demonstrated these differences in DFS to be

219 significant ( $p<0.001$; Fig. 3B). The median DFS times for patients with CARs of $\geq 0.195$ and

$220<0.195$ were 24 (95\% CI [15-33]) and 86 (95\% CI [62-110]) months, respectively. 


\section{Discrimination ability of CAR in subgroup analysis}

223 CAR was significantly correlated with OS in the subgroups of patients with early- and late-stage

224 disease (HR $=7.06,95 \%$ CI [2.58-19.29], $p<0.001 ; \mathrm{HR}=2.97,95 \%$ CI [1.86-4.75], $p<0.001$,

225 respectively. Fig. 4). Nevertheless, CAR was not significantly associated with OS in the

226 subgroup of patients with lymph node metastasis without ENE.

227

\section{Nomogram models}

229 To improve OSCC survival prediction, we established a multivariate nomogram model

230 consisting of CAR, TNM stage, and several clinicopathological factors (Fig. 5A). Figure 5B

231 presents nomogram calibration plots for 3-year OS prediction, and Figure 5C shows the

232 calibration plots for predicting 5-year OS probabilities. The c-index was 0.801 for the nomogram

233 incorporating CAR and clinicopathological prognosticators, higher than that of the nomograms

234 consisting of clinical factors without CAR (0.759) or TNM staging alone (0.685).

235

\section{DISCUSSION}

237 CAR is a strong prognostic indicator for various cancer types. Our literature review revealed that

238 our patient series is the largest thus far and that our study is the first to develop prognostic

239 nomogram model incorporating CAR in patients with OSCC who underwent curative surgery.

240 By integrating preoperative CAR and diverse prognostic factors, we developed a prognostic

241 nomogram to improve survival prediction for patients with OSCC after surgery. The

242 preoperative nomogram's primary advantage is that it can predict individualized 3- and 5-year

243 survival, thereby helping surgeons to identify patients who are likely to benefit from extensive

244 surgery and multidisciplinary management. Nomograms have been used as prognostic adjuncts 
245 for several types of cancer; thus, they play a major role in personalized oncology medicine (Kao

246 et al., 2018, Kattan et al., 1998, Li et al., 2016). In the present study, a high CAR was strongly

247 associated with a more advanced disease stage, lymph node metastasis with ENE, DOI $>10 \mathrm{~mm}$,

248 need for adjuvant therapy, and shorter survival. In addition, the multivariate analysis results

249 revealed high CAR as an independent predictor of lower OS and DFS probability, and only CAR

250 and NLR were significant inflammation-based prognostic biomarkers. ROC curve analysis

251 results also suggested that, compared with CRP, mGPS, NLR, and PLR, CAR has superior

252 discriminatory ability for predicting survival in patients with OSCC. In addition, the subgroup

253 analysis of CAR by disease stage confirmed the prognostic value of CAR. Numerous factors

254 affect cancer treatment outcomes. When making decisions in clinical practice, physicians often

255 consider prognostic factors not involved in TNM staging. The majority of such factors are

256 excluded from the TNM staging system because they cannot be used to independently predict

257 survival outcomes in multivariate models. However, many indicators of OSCC with this

258 limitation potentially influence each other; therefore, omitting them from a staging system may

259 reduce the accuracy of survival prediction. Our study established a multivariate nomogram that

260 integrates clinicopathological variables, including CAR, into the conventional TNM staging

261 system and generates individual probabilities of survival outcomes._Compared with that of the

262 nomogram excluding CAR (0.759), the c-index of the model with CAR was higher (0.801),

263 suggesting the informativeness of CAR in OSCC survival prediction. The c-indices of both

264 nomograms incorporating clinicopathological factors with and without CAR were higher than

265 that of the nomogram based on TNM staging alone (0.684). The current results support our

266 expectation that preoperative CAR is a valuable biomarker for predicting survival outcomes in

267 patients with OSCC. 
268 Systemic inflammation response possibly has a role in OSCC pathogenesis and progression.

269 OSCC leads to interleukin 6 production, and this potentially promotes CRP synthesis in the liver

270 (St John et al., 2004) and play a role as an autocrine tumor growth factor to enhance oral cancer

271 progression (Duffy et al., 2008). Cancer cell invasion and tumor necrosis can positively

272 upregulate systemic inflammatory response, and the inflammatory marker CRP has been

273 previously determined to exhibit an association with survival outcomes in OSCC patients (Huang

274 et al., 2012). In addition, as an indicator of chronic malnutrition, hypoalbuminemia was

275 associated with increased wound infection risk and poor prognosis in patients with head and neck

276 cancer (Danan et al., 2016). Consistent with previous results (Hwang et al., 2015), the current

277 study results indicate a negative correlation between preoperative CRP and albumin levels. This

278 relationship may in part be explained the systemic inflammation-related decreases in albumin

279 levels synthesized in hepatic cells (Don and Kaysen, 2004). Systemic inflammation followed by

280 a decrease in serum albumin levels may lead to sarcopenia, nutritional deficiency, and

281 subsequent poor performance. All these factors could have adverse effects on head and neck

282 cancer prognosis (Bano et al., 2017).

283 Various prognostic prediction models, evaluated by peripheral blood cell counts and systemic

284 inflammatory mediators, have been developed to stratify OSCC patients for optimal treatment. In

285 OSCC patients, mGPS is a potential independent prognostic factor of cancer-specific survival

286 and OS (Farhan-Alanie, McMahon, \& McMillan, 2015), and preoperative circulating CRP levels

287 are associated with pathological aggression and survival outcomes (Chen et al., 2011). Recent

288 meta-analyses have concluded that elevated pretreatment NLR and PLR demonstrated an

289 association with poor prognosis in patients with head and neck cancer (Takenaka et al., 2018a,

290 Yang et al., 2019). In our executed study, CAR demonstrated a discrimination ability superior to

291 that of other scores based on inflammation, namely CRP, mGPS, NLR, and PLR, in resectable 
292 OSCC patients - consistent with findings reported by previous research executed among patients

293 with other types of cancer (Liu et al., 2015, Wei et al., 2015). Park et al. also found that in a

294 long-term follow-up, the AUCs of CAR were consistently higher than the AUCs of the other

295 inflammation-based prognostic scores, and CAR was the only significant prognostic indicator in

296 patients with OSCC after long-term evaluation (Park, Kim, \& Kim, 2016). This may partly be

297 explained by preoperative CAR being a straightforward ratio with a continuous range of values;

298 by contrast, mGPS comprises dichotomized variables that are inherently qualitative with

299 discontinuous values. Notably, the present study discovered that the AUCs of CAR and mGPS,

300 calculated using the CRP and albumin levels, were higher than those of NLR and PLR, which are

301 common prognostic indicators in OSCC (Jariod-Ferrer et al., 2019). These results suggest that

302 the prognostic predictive ability is higher with the CRP-based prognostic score than with the

303 peripheral blood cell count-based prognostic scores in patients with OSCC; CAR integrates the

304 patterns of systemic inflammation and host nutritional status, thus enabling it to more effectively

305 reveal prognostic outcomes.

306 In the present study, we assumed the CAR cutoff of 0.195 may be useful for predicting OS

307 and DFS in patients with OSCC, and this index may have a cutoff specific for primary tumors at

308 different sites (He et al., 2016, Kuboki et al., 2019, Park, Kim, \& Kim, 2016, Yu et al., 2017).

309 Among all studies on head neck cancers, Wang et al. reported the highest cutoff CAR value of

3100.525 as a prognostic indicator in patients with OSCC; their study, however, included high

311 proportions of patients aged $>60$ years and with advanced disease stage (Wang et al., 2019). A

312 recent study on CAR's prognostic value in enrolled patients with advanced hypopharyngeal

313 cancer, two-thirds of whom were aged $>65$ years, also suggested a relatively high CAR cutoff of

3140.32 to be useful for predicting prognosis (Kuboki et al., 2019). Yu et al. found a CAR of 0.047

315 for predicting the laryngeal cancer prognosis; they enrolled a large proportion of patients with 
316 early-stage laryngeal cancer and relatively young population (Yu et al., 2017). On the basis of

317 these study results, the primary tumor site, cancer stage, age distribution of the study cohort, and

318 albumin physiological decrease with aging may all account for the different cutoffs of CAR in

319 head and neck cancer patients.

320 This study's strengths are twofold: (1) we included a relatively large cohort of patients with

321 resectable OSCC and long follow-up period. (2) Preoperative CAR is a highly accessible

322 biological marker that could be applied in daily clinical practice because CAR measurement is

323 easy and noninvasive and causes no additional burden to the patients. However, this study also

324 has two limitations: (1) The retrospective study design has its inherent limitations. Moreover,

325 studies performed at a single center may lead to selection bias. (2) This study investigated

326 preoperative CAR, a measure that may be affected by such factors as undetected infection and

327 cancer-related inflammation. This warrants further investigation of mechanisms specifically

328 underlying the prognostic value of CAR. Finally, although our results suggest that CAR has

329 prognostic value in patients with resectable OSCC, large multi-institue prospective studies are 330 warranted.

332 CONCLUSION

333 Preoperative CAR was found to be an independent prognostic factor regarding OS and DFS in 334 OSCC patients treated with curative surgery, and it has superior discrimination ability to other 335 examined prognostic scores based on inflammation. Higher CAR was significantly correlated 336 with a variety of poor prognosticators. The established multivariate nomogram model that 337 incorporated preoperative CAR and clinicopathological factors into the current TNM staging 338 system might strengthen the accuracy of prognostic prediction for OSCC patients. Due to its 339 simplicity and high availability, CAR can be used as an objective and noninvasive biomarker for 
340 prognostication of OSCC patients undergoing curative surgery and help clinical physician to

341 recognize patients at high risk.

342

343 ACKNOWLEDGEMENTS

344 The authors thank all the members of the HIE lab, Chang Gung Memorial Hospital, for their

345 invaluable help.

346

347

348

349

350

351

352

353

354

355

356

357

358

359

360

361

362

363

364

365

366

\section{References}

Adel M, Liao CT, Lee LY, Hsueh C, Lin CY, Fan KH, Wang HM, Ng SH, Lin CH, Tsao CK +8 more. 2016. Incidence and Outcomes of Patients With Oral Cavity Squamous Cell Carcinoma and Fourth Primary Tumors: A Long-term Follow-up Study in a Betel Quid Chewing Endemic Area. Medicine (Baltimore) 95:e2950

\section{Bano G, Trevisan C, Carraro S, Solmi M, Luchini C, Stubbs B, Manzato E, Sergi G,} Veronese N. 2017. Inflammation and sarcopenia: A systematic review and metaanalysis. Maturitas $\mathbf{9 6 : 1 0 - 1 5}$

Brown DJ, Milroy R, Preston T, McMillan DC. 2007. The relationship between an inflammation-based prognostic score (Glasgow Prognostic Score) and changes in serum biochemical variables in patients with advanced lung and gastrointestinal 
367

368

369

370

371

372

373

374

375

376

377

378

379

380

381

382

383

384

385

386

387

388

389

390

391

392

393

394

395

396

397

398

399

400

401

402

cancer. J Clin Pathol 60:705-708

Chen HH, Chen IH, Liao CT, Wei FC, Lee LY, Huang SF. 2011. Preoperative circulating Creactive protein levels predict pathological aggressiveness in oral squamous cell carcinoma: a retrospective clinical study. Clin Otolaryngol 36:147- 153

Crumley AB, McMillan DC, McKernan M, McDonald AC, Stuart RC. 2006. Evaluation of an inflammation-based prognostic score in patients with inoperable gastrooesophageal cancer. Br J Cancer 94:637-641

Danan D, Shonka DC, Jr., Selman Y, Chow Z, Smolkin ME, Jameson MJ. 2016. Prognostic value of albumin in patients with head and neck cancer. Laryngoscope 126:1567-1571

Don BR, and Kaysen G. 2004. Serum albumin: relationship to inflammation and nutrition. Semin Dial 17:432-437

Duffy SA, Taylor JM, Terrell JE, Islam M, Li Y, Fowler KE, Wolf GT, Teknos TN. 2008. Interleukin- 6 predicts recurrence and survival among head and neck cancer patients. Cancer 113:750-757

Dutta S, Crumley AB, Fullarton GM, Horgan PG, McMillan DC. 2011. Comparison of the prognostic value of tumour- and patient-related factors in patients undergoing potentially curative resection of oesophageal cancer. World J Surg 35:1861-1866

Farhan-Alanie OM, McMahon J, McMillan DC. 2015. Systemic inflammatory response and survival in patients undergoing curative resection of oral squamous cell carcinoma. $\mathrm{Br} J$ Oral Maxillofac Surg 53:126-131

Gellrich NC, Handschel J, Holtmann H, Kruskemper G. 2015. Oral cancer malnutrition impacts weight and quality of life. Nutrients 7:2145-2160

Harrell FE, Jr., Lee KL, Mark DB. 1996. Multivariable prognostic models: issues in developing models, evaluating assumptions and adequacy, and measuring and reducing errors. Stat Med 15:361-387

He S, Wang Y, Chen H, Yang L, Liang S, Lu L, Chen Y. 2016. C-Reactive Protein/Albumin Ratio (CAR) as a Prognostic Factor in Patients with Non-Metastatic Nasopharyngeal Carcinoma. J Cancer 7:2360-2366

Huang SF, Wei FC, Liao CT, Wang HM, Lin CY, Lo S, Huang JJ, Chen IH, Kang CJ, Chien HT, Chen HH. 2012. Risk stratification in oral cavity squamous cell carcinoma by preoperative CRP and SCC antigen levels. Ann Surg Oncol 19:3856-3864

Hwang JC, Jiang MY, Lu YH, Wang CT. 2015. Precedent fluctuation of serum hs-CRP to albumin ratios and mortality risk of clinically stable hemodialysis patients. PLoS One 10:e0120266

Ishizuka M, Nagata H, Takagi K, Iwasaki Y, Shibuya N, Kubota K. 2016. Clinical Significance of the C-Reactive Protein to Albumin Ratio for Survival After Surgery for 
403

404

405

406

407

408

409

410

411

412

413

414

415

416

417

418

419

420

421

422

423

424

425

426

427

428

429

430

431

432

433

434

435

436

437

438

Colorectal Cancer. Ann Surg Oncol 23:900-907

Jariod-Ferrer UM, Arbones-Mainar JM, Gavin-Clavero MA, Simon-Sanz MV, Moral-Saez I, Cisneros-Gimeno AI, Martinez-Trufero J. 2019. Are Comorbidities Associated With Overall Survival in Patients With Oral Squamous Cell Carcinoma? J Oral Maxillofac Surg 77:1906-1914

Kao HK, Lofstrand J, Loh CY, Lao WW, Yi JS, Chang YL, Chang KP. 2018. Nomogram based on albumin and neutrophil-to-lymphocyte ratio for predicting the prognosis of patients with oral cavity squamous cell carcinoma. Sci Rep 8:13081

Kattan MW, Eastham JA, Stapleton AM, Wheeler TM, Scardino PT. 1998. A preoperative nomogram for disease recurrence following radical prostatectomy for prostate cancer. $J$ Natl Cancer Inst 90:766-771

Kuboki A, Kanaya H, Nakayama T, Konno W, Goto K, Nakajima I, Kashiwagi T, Hirabayashi H, Haruna SI. 2019. Prognostic value of C-reactive protein/albumin ratio for patients with hypopharyngeal and laryngeal cancer undergoing invasive surgery involving laryngectomy. Head Neck 41:1342-1350

Li Y, Jia H, Yu W, Xu Y, Li X, Li Q, Cai S. 2016. Nomograms for predicting prognostic value of inflammatory biomarkers in colorectal cancer patients after radical resection. Int $J$ Cancer 139:220-231

Liao CT, Wallace CG, Lee LY, Hsueh C, Lin CY, Fan KH, Wang HM, Ng SH, Lin CH, Tsao CK, Chen IH, Huang SF, Kang CJ, Yen TC. 2014. Clinical evidence of field cancerization in patients with oral cavity cancer in a betel quid chewing area. Oral Oncol 50:721-731

Liu X, Sun X, Liu J, Kong P, Chen S, Zhan Y, Xu D. 2015. Preoperative C-Reactive Protein/Albumin Ratio Predicts Prognosis of Patients after Curative Resection for Gastric Cancer. Transl Oncol 8:339-345

McMillan DC. 2008. An inflammation-based prognostic score and its role in the nutrition-based management of patients with cancer. Proc Nutr Soc 67:257-262

Oliveira A, Rodriguez-Artalejo F, Lopes C. 2010. Alcohol intake and systemic markers of inflammation--shape of the association according to sex and body mass index. Alcohol Alcohol 45:119-125

Park HC, Kim MY, Kim CH. 2016. C-reactive protein/albumin ratio as prognostic score in oral squamous cell carcinoma. J Korean Assoc Oral Maxillofac Surg 42:243-250

Read JA, Choy ST, Beale PJ, Clarke SJ. 2006. Evaluation of nutritional and inflammatory status of advanced colorectal cancer patients and its correlation with survival. Nutr Cancer 55:78-85

Shafique K, Mirza SS, Vart P, Memon AR, Arain MI, Tareen MF, Haq ZU. 2012. Areca nut 
439

440

441

442

443

444

445

446

447

448

449

450

451

452

453

454

455

456

457

458

459

460

461

462

463

464

465

466

467

468

469 chewing and systemic inflammation: evidence of a common pathway for systemic diseases. J Inflamm (Lond) 9:22

St John MA, Li Y, Zhou X, Denny P, Ho CM, Montemagno C, Shi W, Qi F, Wu B, Sinha U, Jordan R, Wolinsky L, Park NH, Liu H, Abemayor E, Wong DT. 2004. Interleukin 6 and interleukin 8 as potential biomarkers for oral cavity and oropharyngeal squamous cell carcinoma. Arch Otolaryngol Head Neck Surg 130:929-935

Takenaka Y, Oya R, Kitamiura T, Ashida N, Shimizu K, Takemura K, Yamamoto Y, Uno A. 2018. Platelet count and platelet-lymphocyte ratio as prognostic markers for head and neck squamous cell carcinoma: Meta-analysis. Head Neck 40:2714-2723

Takenaka Y, Oya R, Kitamiura T, Ashida N, Shimizu K, Takemura K, Yamamoto Y, Uno A. 2018. Prognostic role of neutrophil-to-lymphocyte ratio in head and neck cancer: A meta-analysis. Head Neck 40:647-655

Wang Q, Song X, Zhao Y, He Q, Shi M, Xu P, Ni S, Chen Y, Lin J, Zhang L. 2019. Preoperative high c-reactive protein/albumin ratio is a poor prognostic factor of oral squamous cell carcinoma. Future Oncol 15:2277-2286

Wei XL, Wang FH, Zhang DS, Qiu MZ, Ren C, Jin Y, Zhou YX, Wang DS, He MM, Bai L, Wang F, Luo HY, Li YH, Xu RH. 2015. A novel inflammation-based prognostic score in esophageal squamous cell carcinoma: the $\mathrm{C}$-reactive protein/albumin ratio. $B M C$ Cancer 15:350

Yang L, Huang Y, Zhou L, Dai Y, Hu G. 2019. High pretreatment neutrophil-to-lymphocyte ratio as a predictor of poor survival prognosis in head and neck squamous cell carcinoma: Systematic review and meta-analysis. Head Neck 41:1525-1535

Yu ST, Zhou Z, Cai Q, Liang F, Han P, Chen R, Huang XM. 2017. Prognostic value of the C-reactive protein/albumin ratio in patients with laryngeal squamous cell carcinoma. Onco Targets Ther 10:879-884

Zhang Y, Zeng C, Wei J, Li H, Yang T, Yang Y, Deng ZH, Ding X, Lei G. 2016. Associations of cigarette smoking, betel quid chewing and alcohol consumption with high-sensitivity C-reactive protein in early radiographic knee osteoarthritis: a crosssectional study. BMJ Open 6:e010763 


\section{Table $\mathbf{1}$ (on next page)}

Table 1: Baseline Clinicopathological and Laboratory Characteristics of 326 Patients with OSCC.

Notes: OSCC: oral cavity squamous cell carcinoma; ENE: extracapsular nodal extension; RT: radiotherapy; CCRT: concurrent chemoradiotherapy; mGPS: modified Glasgow prognostic score; CAR: C-reactive protein-to-albumin ratio; NLR: neutrophil-to-lymphocyte ratio; PLR: platelet-to-lymphocyte ratio; SD: standard deviation 
1

\begin{tabular}{lr}
\hline Variable & Characteristics \\
\hline Age (years) & \\
$<65$ & $242(74.2 \%)$ \\
$\geq 65$ & $84(25.8 \%)$
\end{tabular}

Sex

Men

$294(90.2 \%)$

Women

$32(9.8 \%)$

Primary tumor site

Tongue

$126(38.7 \%)$

Buccal mucosa

$104(31.9 \%)$

Gingiva

$43(13.2 \%)$

Retromolar trigone

$20(6.1 \%)$

Lip

$14(4.3 \%)$

Mouth floor

$13(4.0 \%)$

Hard palate

$6(1.8 \%)$

Cigarette smoking

$267(81.9 \%)$

Alcohol consumption

$215(66.0 \%)$

Betel nut chewing

$260(79.8 \%)$

\section{TNM Staging}

I

$71(21.7 \%)$

II

$64(19.6 \%)$

III

$39(11.9 \%)$

IV

$152(46.6 \%)$

pT classification

$\mathrm{T} 1$

$90(27.6 \%)$ 
$\mathrm{T} 2$

T3

$\mathrm{T} 4$

\section{Nodal status}

Metastasis (-), ENE (-)

Metastasis (+), ENE (-)

Metastasis (+), ENE (+)

\section{Cell differentiation}

Well

Moderate

Poor

\section{Depth of invasion $\geq 10 \mathrm{~mm}$}

Yes

No

\section{Adjuvant therapy}

Absent

RT

CCRT

mGPS

0

1 or 2

CAR, median (IQR)

NLR, median (IQR)

PLR, median (IQR)
$96(29.4 \%)$

$$
22(6.7 \%)
$$

$118(36.2 \%)$

$209(64.1 \%)$

$51(15.6 \%)$

$66(20.2 \%)$

$94(28.8 \%)$

$194(59.5 \%)$

$38(11.7 \%)$

$153(46.9 \%)$

$173(53.1 \%)$

$178(54.6 \%)$

$43(13.2 \%)$

$105(32.2 \%)$

$227(69.6 \%)$

$99(30.4 \%)$

$0.08(0.03-0.34)$

$2.37(1.73-3.42)$

$114.01(87.60-154.00)$

2 


\section{Table 2 (on next page)}

Table 2: Baseline Clinicopathological Characteristics According to the CAR.

Notes: CAR: C-reactive protein-to-albumin ratio; ENE: extracapsular nodal extension; RT: radiotherapy; CCRT: concurrent chemoradiotherapy; mGPS: modified Glasgow prognostic score; NLR: neutrophil-to-lymphocyte ratio; PLR: platelet-to-lymphocyte ratio; SD: standard deviation; $\mathbf{C l}$ : confidence interval ${ }^{*}$ the Chi-square test ${ }^{* *}$ the Mann-Whitney $U$ test ( Z-test: NLR: -4.65; PLR: -2.81; Survival in months: -3.28). 


\begin{tabular}{|c|c|c|c|}
\hline \multirow[b]{2}{*}{ Variable } & \multicolumn{2}{|c|}{ Number of patients } & \multirow[b]{2}{*}{$p$ value } \\
\hline & $\begin{array}{l}\text { CAR }<0.195 \\
(n=214)\end{array}$ & $\begin{array}{l}C A R \geq 0.195 \\
(n=112)\end{array}$ & \\
\hline Sex & & & $0.434^{*}$ \\
\hline Men & $191(89.3 \%)$ & $103(92.0 \%)$ & \\
\hline Women & $23(10.7 \%)$ & $9(8.0 \%)$ & \\
\hline Age & & & $0.170^{*}$ \\
\hline$<65$ & $164(76.6 \%)$ & $78(69.6 \%)$ & \\
\hline$\geq 65$ & $50(23.4 \%)$ & $34(30.4 \%)$ & \\
\hline TNM Staging & & & $<0.001^{*}$ \\
\hline I & $57(26.6 \%)$ & $14(12.5 \%)$ & \\
\hline II & $51(23.8 \%)$ & $13(11.6 \%)$ & \\
\hline III & $24(11.2 \%)$ & $15(13.4 \%)$ & \\
\hline IV & $82(38.4 \%)$ & $70(62.5 \%)$ & \\
\hline pT classification & & & $<0.001^{*}$ \\
\hline $\mathrm{T} 1$ & $71(33.2 \%)$ & $19(17.0 \%)$ & \\
\hline $\mathrm{T} 2$ & $69(32.2 \%)$ & $27(24.1 \%)$ & \\
\hline $\mathrm{T} 3$ & $11(5.1 \%)$ & $11(9.8 \%)$ & \\
\hline $\mathrm{T} 4$ & $63(29.4 \%)$ & $55(49.1 \%)$ & \\
\hline Nodal status & & & $<0.001^{*}$ \\
\hline Metastasis (-), ENE (-) & $154(72.0 \%)$ & $55(49.1 \%)$ & \\
\hline Metastasis $(+)$, ENE $(-)$ & $31(14.5 \%)$ & $20(17.9 \%)$ & \\
\hline Metastasis (+), ENE (+) & $29(13.6 \%)$ & $37(33.0 \%)$ & \\
\hline Cell differentiation & & & $0.205^{*}$ \\
\hline Well & $59(27.6 \%)$ & $35(31.3 \%)$ & \\
\hline
\end{tabular}



Moderate
$134(62.6 \%)$
$60(53.6 \%)$
Poor
$21(9.8 \%)$
$17(15.2 \%)$

Depth of invasion $\geq 10 \mathrm{~mm}$

$<0.001^{*}$

No

$134(62.6 \%)$

$39(34.8 \%)$

Yes

$80(37.4 \%)$

$73(65.2 \%)$

Adjuvant therapy

$0.003^{*}$

Absent

$130(60.7 \%)$

$48(42.9 \%)$

RT

$28(13.1 \%)$

$15(13.4 \%)$

CCRT

$56(26.2 \%)$

$49(43.8 \%)$

mGPS

0

$214(100.0 \%)$

$13(11.6 \%)$

1 or 2

$0(0 \%)$

$99(88.4 \%)$

NLR $($ mean \pm SD)

$2.5 \pm 1.4$

$3.5 \pm 2.2$

$<0.001^{* *}$

PLR $($ mean \pm SD)

$119.5 \pm 52.2$

$149.8 \pm 99.8$

$0.005^{* *}$

Survival in months,

mean $(95 \%$ CI)

$48.5(44.8-52.1)$

$39.2(33.2-45.1)$

$0.001^{* *}$ 


\section{Table 3 (on next page)}

Table 3: Univariate and Multivariate Analysis of Poor Prognostic Factors for OS in OSCC Patients.

Notes: OS: overall survival; OSCC: oral cavity squamous cell carcinoma; HR: Hazard ratio;

Cl: confidence interval; ENE: extracapsular nodal extension; RT: radiotherapy; CCRT: concurrent chemoradiotherapy; mGPS: modified Glasgow prognostic score; CAR: C-reactive protein-to-albumin ratio; NLR: neutrophil-to-lymphocyte ratio; PLR: platelet-to-lymphocyte ratio 
1

Variable

Univariate analysis

Multivariate analysis

5-year OS

HR (95\% CI)

$p$ value

HR $(95 \% \mathrm{CI})$

$p$ value

Sex

Women

$77.3 \%$

Reference

Reference

Men

$67.7 \%$

$1.595(0.737-3.452)$

0.236

$1.069(0.455-2.516)$

0.878

Age (years)

$<65$

$70.1 \%$

Reference

Reference

$\geq 65$

$64.6 \%$

$1.332(0.864-2.054)$

0.194

$1.543(0.926-2.569)$

0.096

\section{pT classification}

$\mathrm{T} 1$

$\mathrm{T} 2$

T3

$\mathrm{T} 4$

\section{Nodal status}

Metastasis (-), ENE (-)

Metastasis (+), ENE (-)

Metastasis (+), ENE (+)

\section{Cell differentiation}

Well

Moderate

Poor

Depth of invasion $\geq 10 \mathrm{~mm}$

$\begin{array}{lll}\text { No } & 76.7 \% & \text { Reference } \\ \text { Yes } & 59.7 \% & 2.108(1.396-3.182)\end{array}$

79.7\% Reference

$60.8 \% \quad 2.033(1.161-3.560)$

$40.4 \%$

$4.405(2.813-6.899)$

$75.7 \%$

$71.2 \%$

$40.6 \%$

$3.911(2.124-7.201)$
Reference

$0.064 \quad 2.182(0.912-4.681)$

0.073

$0.010 \quad 3.206(1.158-8.875)$

0.025

$<0.001 \quad 3.361(1.513-7.465)$

0.003
Reference

$0.013 \quad 1.445(0.796-2.623)$

0.226

$<0.001$

$2.725(1.617-4.593)$

$<0.001$

\section{Adjuvant therapy}




\begin{tabular}{llllll} 
Absent & $76.5 \%$ & Reference & \multicolumn{2}{c}{ Reference } \\
RT & $69.9 \%$ & $1.571(0.834-2.962)$ & 0.162 & $1.577(0.786-3.163)$ & 0.199 \\
CCRT & $55.8 \%$ & $2.363(1.529-3.653)$ & $<0.001$ & $1.173(0.677-2.031)$ & 0.573 \\
GPS & & & & & \\
0 & $77.3 \%$ & Reference & & Reference & \\
1 or 2 & $49.0 \%$ & $3.491(2.325-5.241)$ & $<0.001$ & $0.916(0.418-2.007)$ & 0.827
\end{tabular}

\section{CAR}

\begin{tabular}{llllll}
$<0.195$ & $80.9 \%$ & Reference & \multicolumn{2}{l}{ Reference } \\
$\geq 0.195$ & $46.5 \%$ & $4.397(2.880-6.714)$ & $<0.001$ & $3.614(1.629-8.018)$ & 0.002
\end{tabular}

\section{NLR}

$<4.505$

$75.8 \%$

Reference

Reference

$\geq 4.505$

$22.2 \%$

$4.515(2.911-7.003)$

$<0.001$

$2.271(1.263-4.085)$

0.006

PLR

$<165.85$

$75.9 \%$

Reference

Reference

$\geq 165.85$

$37.8 \%$

$3.338(2.176-5.122)$

$<0.001$

$1.576(0.874-2.843)$

0.131 


\section{Table 4 (on next page)}

Table 4: Univariate and Multivariate Analysis of Poor Prognostic Factors for DFS in OSCC Patients.

Notes: DFS: disease-free survival; OSCC: oral cavity squamous cell carcinoma; HR: Hazard ratio; Cl: confidence interval; ENE: extracapsular nodal extension; RT: radiotherapy; CCRT: concurrent chemoradiotherapy; mGPS: modified Glasgow prognostic score; CAR: C-reactive protein-to-albumin ratio; NLR: neutrophil-to-lymphocyte ratio; PLR: platelet-to-lymphocyte ratio 
1

\begin{tabular}{llllll}
\hline & & & \multicolumn{2}{l}{ Univariate analysis } & Multivariate analysis \\
\cline { 3 - 6 } Variable & 5-year DFS & HR $(95 \% \mathrm{CI})$ & $p$ value & HR $(95 \% \mathrm{CI})$ & $p$ value \\
\hline
\end{tabular}

Sex
Women
49.9\% Reference
Reference
Men
$68.5 \% \quad 1.533(0.844-2.785)$
0.160
$1.054(0.532-2.087)$
0.881

Age (years)

$<65$

$\geq 65$

\section{pT classification}

$\mathrm{T} 1$

$\mathrm{T} 2$

$\mathrm{T} 3$

$\mathrm{T} 4$

\section{Nodal status}

Metastasis (-),ENE (-)

Metastasis (+),ENE (-)

Metastasis (+),ENE (+)

\section{Cell differentiation}

Well

Moderate

Poor

$\begin{array}{ll}51.6 \% & \text { Reference } \\ 52.9 \% & 0.935(0.646-1.352)\end{array}$

$62.8 \% \quad$ Reference

$57.6 \% \quad 1.031(0.647-1.643)$

$47.6 \%$

1.535 (0.777-3.030)

$38.8 \%$

$1.983(1.311-3.001)$

$60.2 \%$

Reference

$50.8 \%$

$1.261(0.791-2.009)$

2.713 (1.884-3.907)

$<0.001$

$27.1 \%$

$49.9 \%$

Reference

$57.4 \%$

0.946 (0.656-1.365)

$33.2 \%$

$2.029(1.240-3.319)$

$55.9 \% \quad$ Reference

$47.6 \%$

$1.379(0.999-1.902)$

Yes

\section{Adjuvant therapy}

Absent

RT

CCRT

mGPS

0

\section{$55.5 \% \quad$ Reference}

$55.8 \%$

$1.052(0.635-1.744)$

$44.4 \%$

$1.416(1.001-2.003)$

$57.5 \% \quad$ Reference
0.330

0.898

0.217

0.001

0.766

0.005

0.050

Reference

Reference

$1.041(0.686-1.580)$

0.849

Reference

1.147 (0.695-1.893)

0.592

$1.468(0.677-3.181)$

0.331

$1.881(1.061-3.335)$

0.031

Reference

$1.285(0.779-2.121)$

0.326

$2.279(1.487-3.494)$

$<0.001$

Reference

$1.058(0.710-1.577) \quad 0.780$

$2.104(1.206-3.672)$

0.009

$0.808(0.512-1.277)$

0.361

Reference

$0.843 \quad 0.911(0.530-1.566)$

0.737

0.049

$0.745(0.485-1.143)$

0.178 
1 or 2

CAR

$<0.195$

$\geq 0.195$

NLR

$<4.505$

$\geq 4.505$

\section{PLR}

$<165.85$

$\geq 165.85$
$39.1 \%$

$60.1 \%$

$36.8 \%$

$57.5 \%$

$16.7 \%$

$56.6 \%$

Reference

$32.9 \%$

Reference
$1.867(1.344-2.592)$

$2.081(1.506-2.875)$

$<0.001$

$1.917(1.051-3.863)$

0.029

2

3

4

5

6 
Figure 1

Figure 1: The scatter plot of the correlation between pretreatment CRP and albumin levels in patients with OSCC.

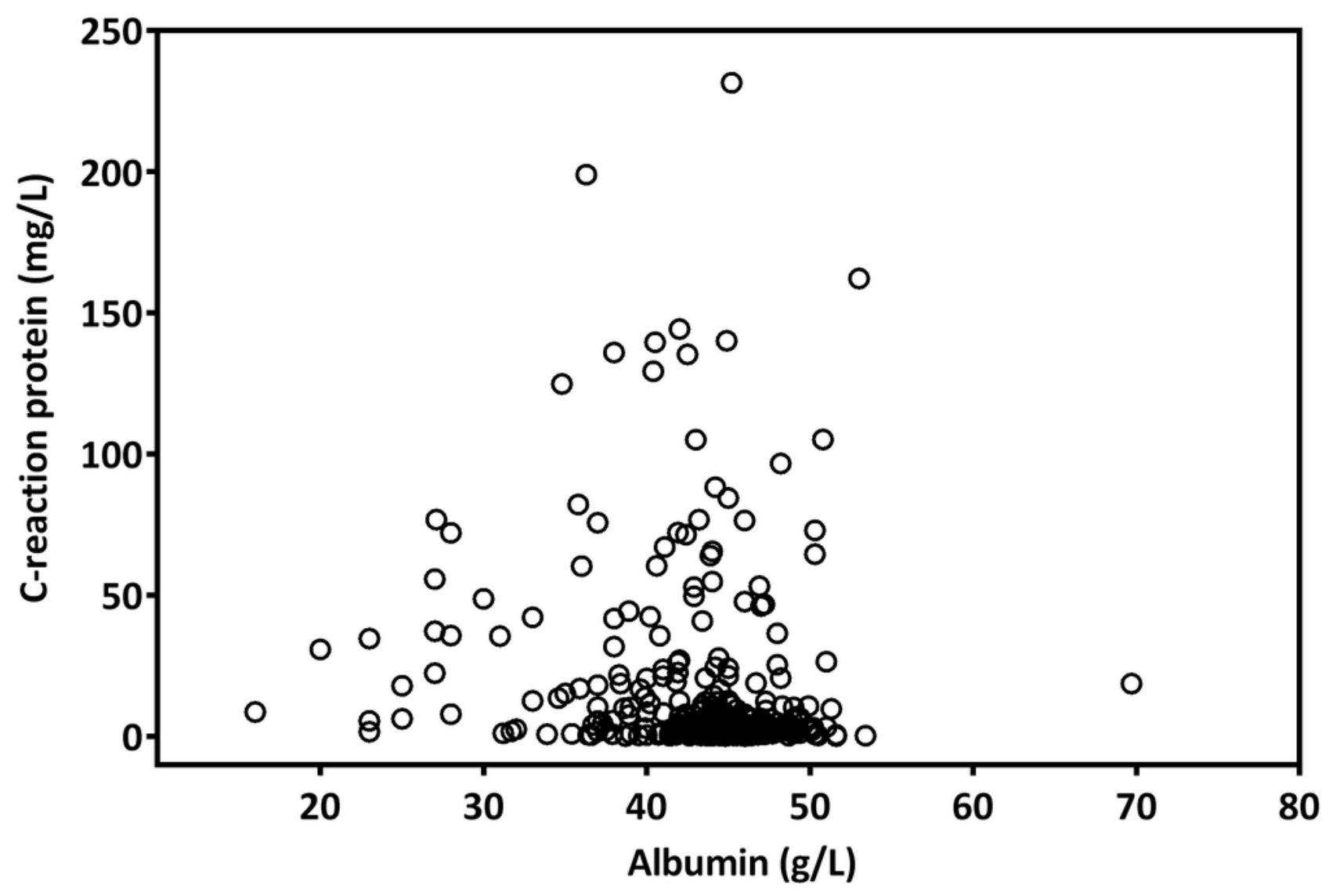


Figure 2

Figure 2: ROC curves were applied to compare predictive ability of four inflammationbased prognostic scores.

The AUC for CAR was larger (0.718) than that for CRP (0.705), mGPS (0.679), NLR (0.621), and PLR (0.610). 


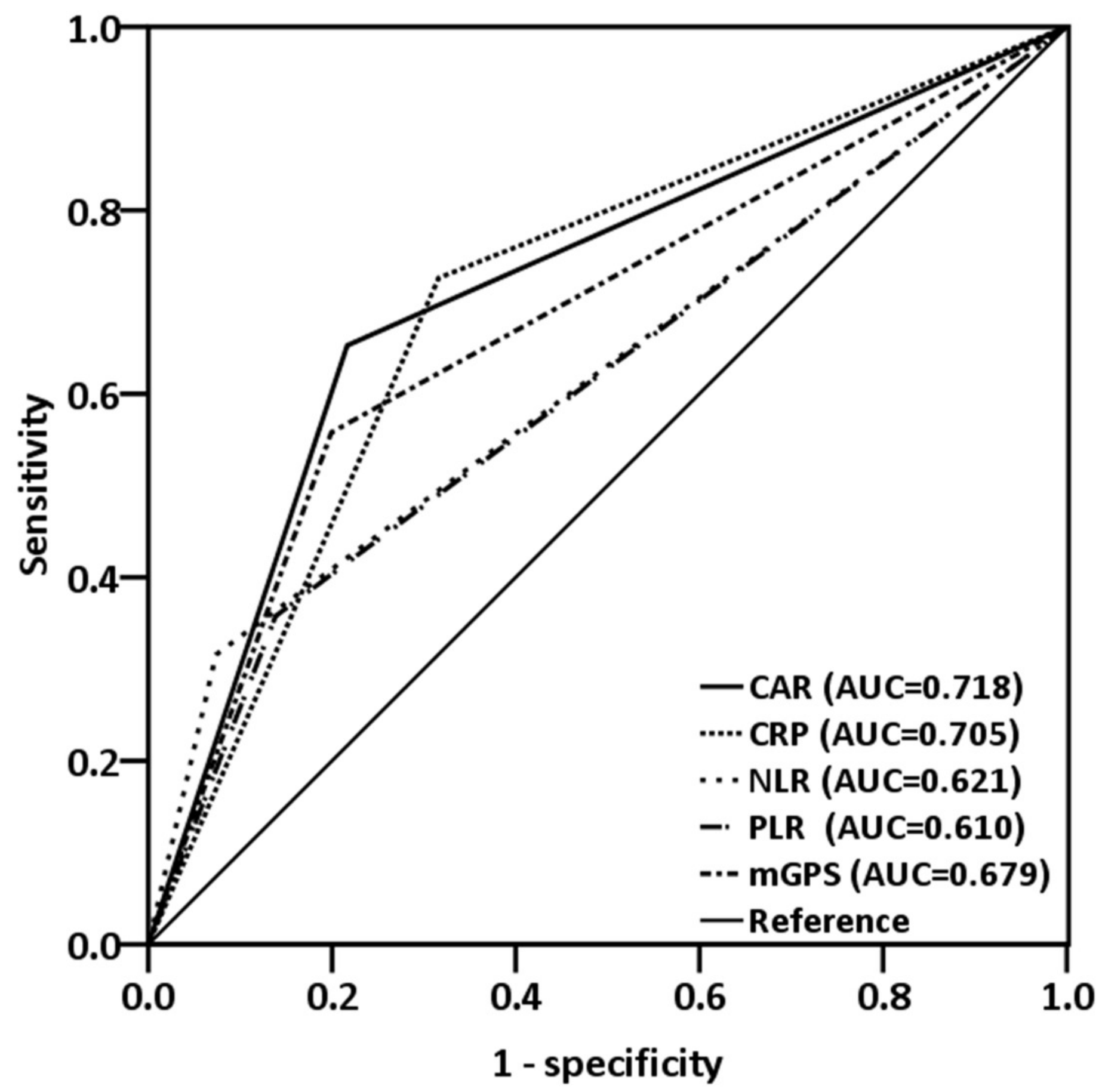


Figure 3

Figure 3: Kaplan-Meier estimates of 5-year OS (A) and DFS (B) according to the optimal pretreatment CAR cutoffs.

(A)

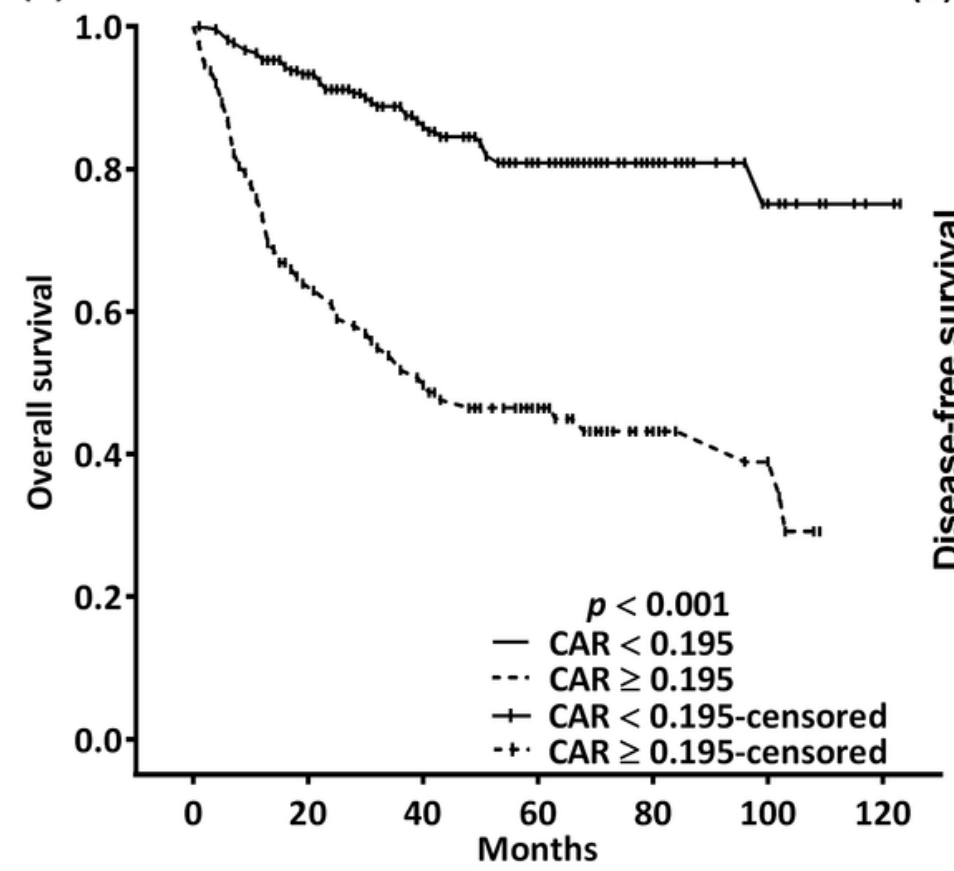

(B)

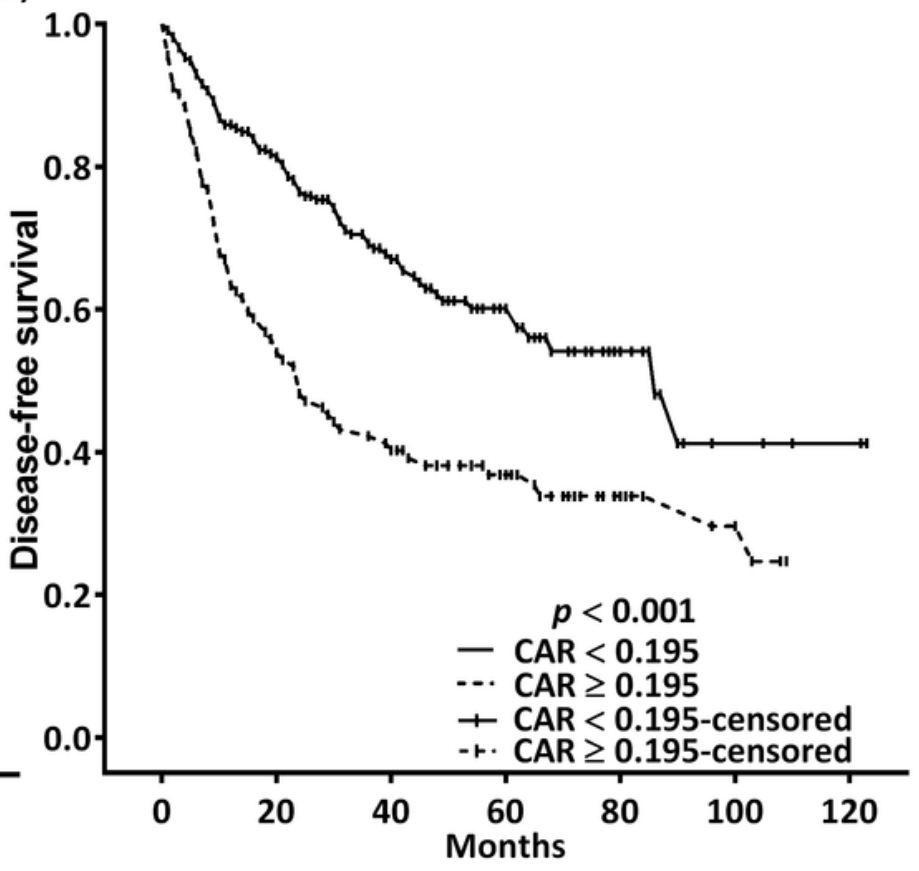




\section{Figure 4}

Figure 4: Hazard ratios (HRs) of CAR in different patient subgroups identified by neck metastatic lymphadenopathy and TNM staging. HRs $>1.0$ indicated a worse outcome.

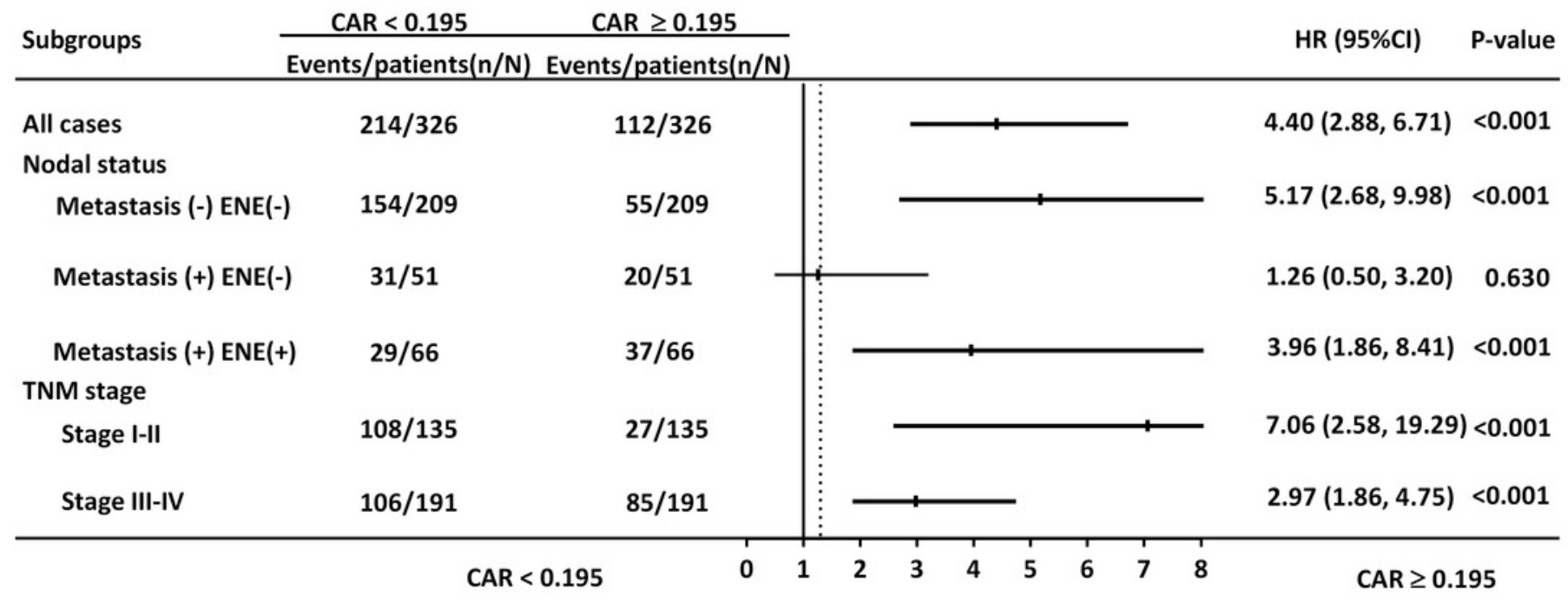




\section{Figure 5}

Figure. 5 (A) Nomogram for OS prediction for patients with OSCC. A line runs vertically from each parameter to the uppermost points. Summing the scores for each parameter provides the total score, which can be translated into survival probabilities along

Abbreviations: ENE, extracapsular nodal extension; DOI, depth of invasion; W-D, well differentiated squamous cell carcinoma; M-D, moderately differentiated squamous cell carcinoma; P-D, poorly differentiated squamous cell carcinoma; CAR $=$ C-reactive protein to albumin ratio.

(A)

Points
Sex
Age
Stage
ENE
Cell differentiation
DOI
CAR
Total Points
3 year survival
5 year survival

(B)

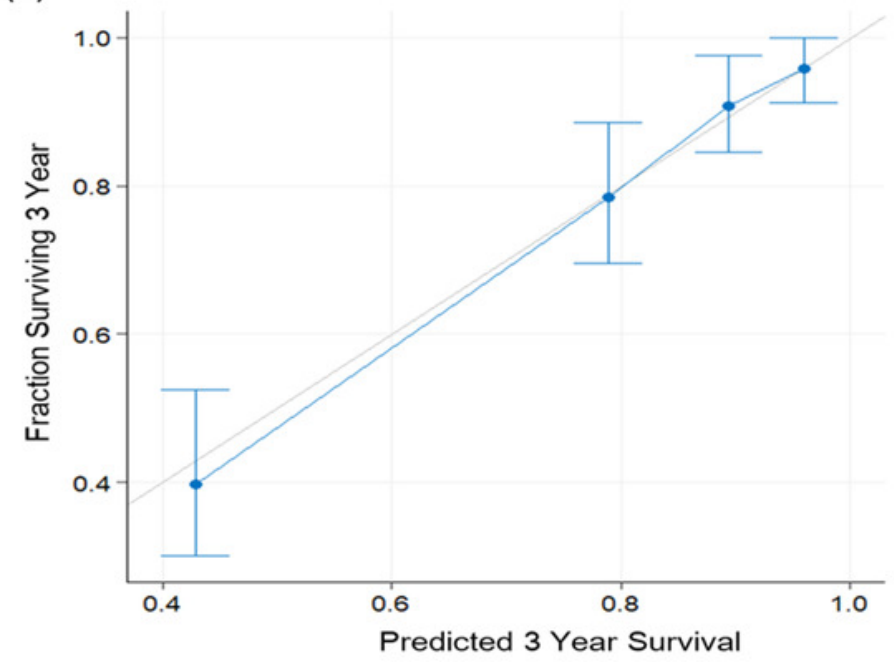

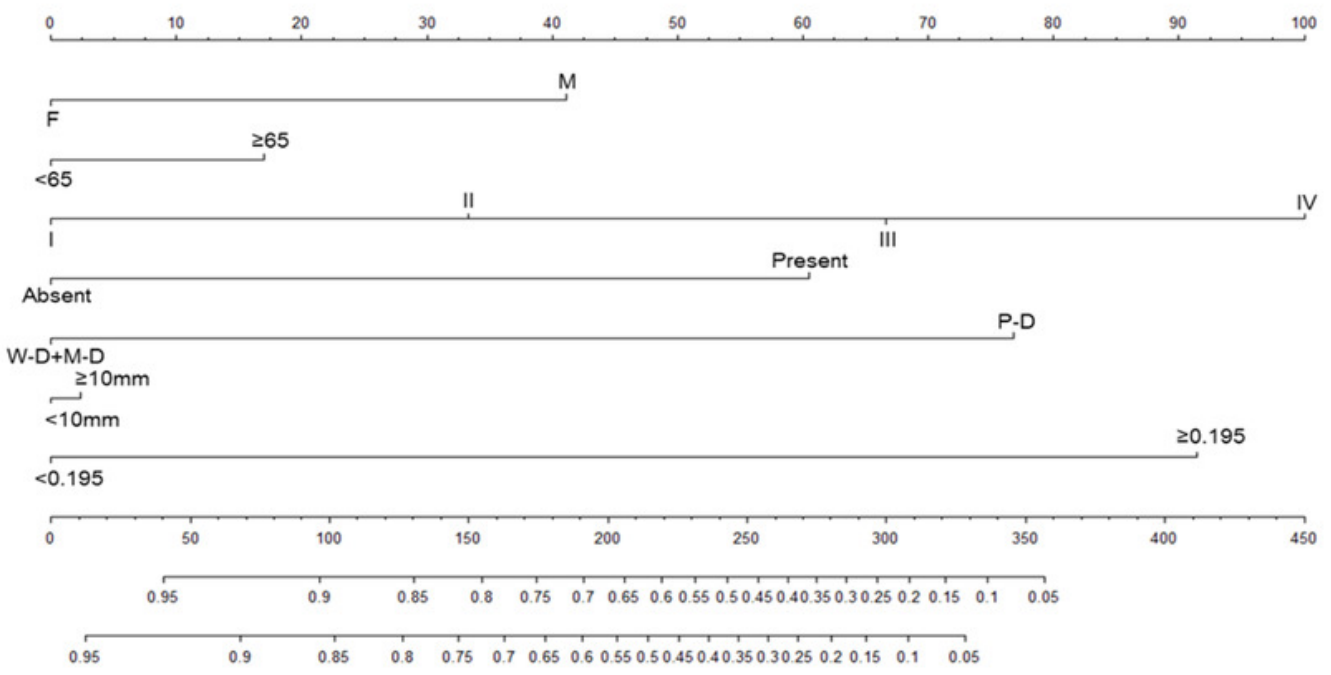

(C)

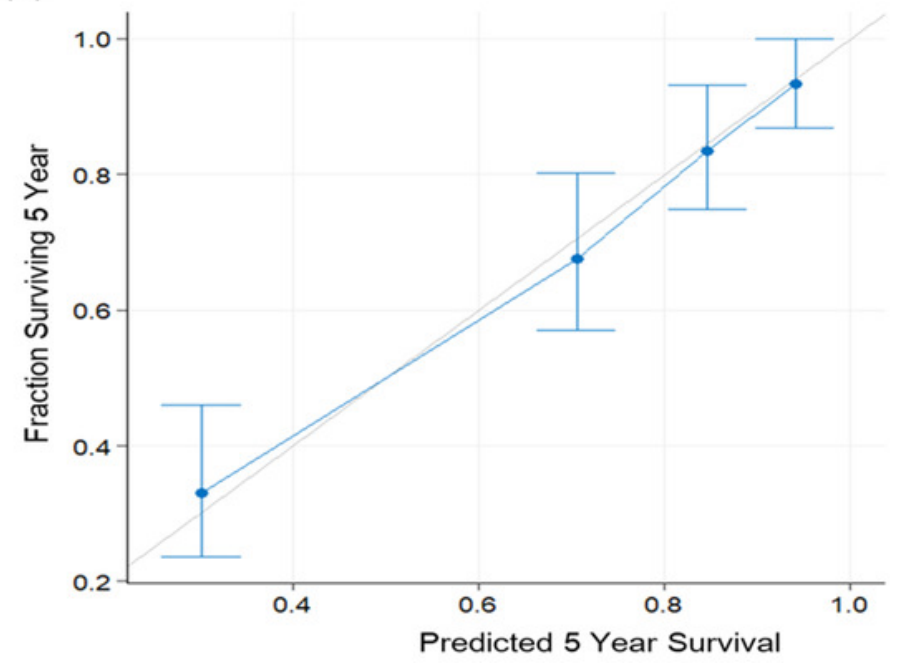

